\title{
Minimax Frequency Domain Performance and Robustness Optimization of Linear Feedback Systems
}

\author{
HUIBERT KWAKERNAAK, FELLOW, IEEE
}

\begin{abstract}
It is shown that feedback system design objectives, such as disturbance attenuation and rejection, power and bandwidth limitation, and robustness, may be expressed in terms of required bounds of the sensitivity function and its complement on the imaginary axis. This leads to a minimax frequency domain optimization problem, whose solution is reduced to the solution of a polynomial equation.
\end{abstract}

\section{INTRODUCTION ,}

$\mathbf{F}$ EEDBACK systems are designed to fulfill a number of purposes. Among them are disturbance attenuation and rejection, good command response, and robustness. Restricted plant power and sensitivity to sensor noise form important limitations. Optimization is a powerful technique to deal with design problems with conflicting objectives. It therefore is little wonder that the optimization of control systems, particularly linear control systems, is a subject of long standing.

Inspired by the work of Wiener, much of the effort has been directed towards the minimization of quadratic integral-type criteria, either handled in the frequency domain such as in Wiener optimization, or in the time domain such as in linear optimal control theory. The principal reason for this emphasis on quadratic criteria is the existence of explicit solutions to the corresponding optimization problems and their accessibility for analysis.

Although linear optimal control theory with quadratic criteria is a powerful tool, it has limitations. One of its principal disadvantages is its inability to handle robustness problems. There seems to be no natural way to include robustness requirements as a design objective in quadratic integral criteria.

Much of the recent work on robustness has been focused on frequency domain characterizations [1]-[5]. The results mainly concern multiinput multioutput feedback systems in the configuration of Fig. 1, but the conclusions are of course also valid for single-input single-output systems, which are the subject of this paper. In the block diagram of Fig. $1, H$ is the plant transfer function and $G$ the controller transfer function. The loop gain is denoted as $L(s):=H(s) G(s)$. From this recent work on robustness it is clear that the sensitivity function $S:=1 /(1+L)$ and its complement $T:=1-S=L /(1+L)$ play an important role in assessing the robustness of a closed-loop control system. It has also become evident that good robustness may be obtained if suitable bounds can be imposed on the behavior of $S$ and $T$ on the imaginary axis.

In this connection, an important field of study has been opened up by Zames [6]-[8]. Zames argues that under partial uncertainty about the nature of the disturbances it is necessary to impose bounds on the sensitivity function. This idea leads to an optimization problem that consists of the minimization of a criterion of the form $\sup _{\omega}|S(i \omega) V(i \omega)|$, where $V$ is a suitable

Manuscript received October 19, 1983; revised August 21, 1984, November 21,1984 , and January 14,1985 . This paper is based on a prior submission of July 12, 1982. Paper recommended by Past Associate Editor, B. R. Barmish.

The author is with the Department of Applied Mathematics, Twente University of Technology, Enschede, The Netherlands.

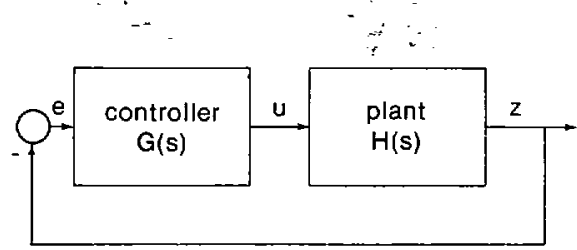

Fig. 1. Control system configuration.

weighting function. The problem, as well as its multivariable version [9], [10], are solved by interpolation theory or functional analysis techniques. It is somewhat surprising that fairly"explicit solutions exist and that no recourse needs be taken to nonlinear programming.

In the present paper it is attempted to take this work a little further and to show that many essential design objectives for linear feedback systems may be translated into required bounds on the sensitivity function $S$ or its complement $T$, or both, on the imaginary axis. These design objectives include disturbance attenuation and rejection, power and bandwidth limitation, and robustness.

Section II of this paper is devoted to a discussion of these subjects. It is concluded that a balance between conflicting design objectives can be achieved by minimizing a criterion of the form

$$
\sup _{\omega}\left[|V(i \omega) S(i \omega)|^{2}+|W(i \omega) T(i \omega)|^{2}\right]
$$

where $V$ and $W$ are suitably chosen weighting functions. As in the work of Safonov, Laub, and Hartmann [11], the criterion involves both $S$ and $T$, but it is a minimax and not an integral type criterion.

The actual solution of the minimax problem is undertaken in Section III. The solution technique differs from that of other authors and is related to the well-known fact in, for instance, statistical decision theory that "equalizers" yield minimax solutions. It is shown that the minimax problem may be reduced to the solution of a polynomial equation.

Section IV, which is quite brief, offers a few comments on the numerical solution of this polynomial equation. Section $\mathrm{V}$ deals with the specialization of the results to the minimization of $\sup _{\omega}|V(i \omega) S(i \omega)|$. Finally, in Section VI some examples are discussed. Three of them illustrate technical points. The fourth shows that application of the design technique to a stock example (the double integrator) leads to a classical design. The conclusions of the paper are summarized in Section VII.

Extension of the results of this paper to multiinput multioutput plants is feasible and is currently under investigation [12]. For single-input single-output systems, the results of the current paper may be generalized to a criterion of the form $\Sigma_{k} \mid V_{k}(i \omega) S(i \omega)+$ $\left.W_{k}(i \omega) T(i \omega)\right|^{2}$, with the $V_{k}$ and $W_{k}$ suitable weighting functions. These results are reported elsewhere [13]. Since the first version of the present paper was written, Verma and Jonckheere [14] published a solution of the problem of minimizing (1.1) based on Helton's work [15], while Francis [16] solved the multivariable version of the problem using functional analysis techniques.

We end this Introduction with some preliminaries. The plant transfer function $H$ and the controller transfer function $G$ are 
written in the form

$$
H(s)=\frac{\psi(s)}{\phi(s)}, G(s)=\frac{\rho(s)}{\sigma(s)}
$$

where $\psi, \phi, \rho$, and $\sigma$ are real polynomials such that $\psi$ and $\phi$ have no common roots. The plant denominator polynomial $\phi$ has degree $n$ and the numerator polynomial $\psi$ degree $m . G$ is not necessarily proper, but we assume that $H(s)$ and $L(s)=H(s) G(s)$ are. Under these assumptions the closed-loop system is welldefined as long as $L_{\infty} \neq-1$, where $L_{\infty}:=\lim _{|s| \rightarrow \infty} L(s)$. When both $\phi$ and $\sigma$ are monic, the closed-loop characteristic polynomial is given by

$$
\chi_{o l}(s) \frac{1+L(s)}{1+L_{\infty}}:+\cdots
$$

where $\chi_{o l}(s):=\phi(s) \sigma(s)$ is the open-loop characteristic polynomial. In the sequel it is convenient to work with the unnormalized characteristic polynomial

$$
\chi_{c l}(s):=\chi_{o l}(s)(1+L(s)) \text {. }
$$

If the closed-loop system is well-defined; this polynomial equals the closed-loop characteristic polynomial within a nonzero constant factor, also when $\phi$ and $\sigma$ are not monic. In terms of polynomials,

$$
\chi_{c l}(s)=\phi(s) \sigma(s)+\psi(s) \rho(s) .
$$

The sensitivity function $S$ and its complement $T$ may be expressed as

$$
S(s)=\frac{\phi(s) \sigma(s)}{\chi_{c l}(s)}, T(s)=\frac{\psi(s) \rho(s)}{\chi_{c l}(s)}
$$

\section{Performance and Robustness Optimization by Optimal BOUNDING}

In this section it will first be shown that many essential control system design requirements can be translated into bounds on the behavior of the sensitivity function $S$ and its complement $T$ on the imaginary axis. The next step in the argument is to minimize these bounds. This leads to a minimax frequency domain optimization problem, whose solution is discussed in the next section. The design aspects considered in the present section are disturbance attenuation, disturbance rejection, power limitation-which is directly related to bandwidth limitation-robustness, and command response.

In the following, the discussion will be confined to closed-loop systems that have all their poles in the closed left-half plane. Controllers that result in control systems not satisfying this requirement are not admissible.

Disturbance Attenuation: For good disturbance attenuation, the sensitivity function $S$ of the closed-loop system should have the property that $|S(i \omega)|$ is small over the frequency range of the disturbances. It has been convincingly argued by Zames [6] that this can be achieved by requiring that $|S(i \omega) V(i \omega)|$ is uniformly small for all $\omega$, where $V$ is a suitable weighting function reflecting the available information about the frequency content of the disturbances. Thus, for good disturbance attenuation we need to be ensured of a sufficiently small upper bound on $|S(i \omega) V(i \omega)|$.

Disturbance Rejection: Consider a disturbance (entering at the output of the plant) of the form $t^{j} e^{\lambda t}, j=0,1, \cdots, k-1$, with $\lambda$ a given complex number such that $\operatorname{Re}(\lambda) \geq 0$, and $k$ a given integer. It is said that the closed-loop control system rejects this disturbance if the response of the controlled output to this disturbance eventually vanishes. A necessary and sufficient condition for disturbance rejection is that $S$ has a zero of multiplicity $k$ at $s=\lambda$.

It is mandatory to have rejection of disturbances that are plant generated. These are disturbances that result from nonzero initial conditions of the plant, and are of the indicated type with $\lambda$ any plant pole in the closed right-half plane and $k$ its multiplicity. Since $S(s)=\phi(s) \sigma(s) / \chi_{c l}(s), S$ always has zeros, with the correct multiplicity, at the open-loop plant poles in the open right-half plane; by admissibility such poles can never cancel against roots of $\chi_{c l}$. We can make sure that $S$ has zeros, with the correct multiplicity, at the plant poles on the imaginary axis by requiring that $|S(i \omega) V(i \omega)|$ is bounded, where $V$ has poles at all open-loop plant poles on the imaginary axis with corresponding multiplicity.

If $V$ also has poles at other locations on the imaginary axis, rejection is achieved for the corresponding disturbances as well. This may be useful for vibration isolation (rejection of harmonic disturbances of a fixed frequency), but is particularly important for obtaining rejection of constant disturbances by letting $V$ have a pole at $s=0$. In particular, if $V$ has a pole at $s=0$ of multiplicity $k,|S(i \omega) V(i \omega)|$ can only be bounded if $S$ has a zero at 0 of multiplicity $k$, which means that the closed-loop system is of "type- $k$."

Power and Bandwidth Limitation: In the closed-loop system, the transfer function from the disturbances, measurement noise, and command input to the plant input is $T(s) / H(s)$. Limitation of the input power of the plant can therefore be achieved by imposing a bound on $|W(i \omega) T(i \omega)|$, where $W$ is a weighting function that is suitably chosen in relation to the plant transfer function $H$ and the available information about the frequency content of disturbances, measurement noise, and command input.

We record some important considerations in the choice of the weighting function $W$. First, we note that if the plant has pole excess $e \geq 0,1 / H(s)=0\left(s^{e}\right)$ as $|s| \rightarrow \infty$. Therefore, in order to make sure that $|T(i \omega) / H(i \omega)|$ remains bounded as $\omega \rightarrow \infty-$ which certainly is advisable - one should make sure that $W(s)=0\left(s^{e}\right)$ as $|s| \rightarrow \infty$. If $W$ has this property and $|W(i \omega) T(i \omega)|$ is bounded, $T$ necessarily has pole excess $e$ (or more). Since $L(s)=T(s) /(1-$ $T(s)$ ), the loop gain $L$ has the same pole excess $e$ as $T$. Consequently, since $L(s)=H(s) G(s)$, the controller transfer function $G$ necessarily is proper. By letting $W(s)=0\left(s^{p}\right)$ with $p$ $\geq e$ we can in fact impose any desired pole excess $p-e$ on the controller transfer function $G$.

Next, assume that $W(s)=0\left(s^{p}\right)$ for $|s| \rightarrow \infty$, with $p \geq e$. Then that frequency $\omega_{1}$ from which the behavior of $W(i \omega)$ starts to be dominated by $(i \omega)^{p}$ is the frequency from which $T(i \omega)$ starts to decrease as $1 /(i \omega)^{p}$; therefore, $\omega_{1}$ is nothing else than the bandwidth of the closed-loop system. Thus, by a suitable choice of $W$ it is possible to control the closed-loop bandwidth.

Finally, we note that $T(s) / H(s)$ will have poles at the plant zeros unless these are canceled by corresponding zeros of $T$. Because of power limitation, $T(s) / H(s)$ should have no poles on or to the right of the imaginary axis. Since $T(s) / H(s)=\phi(s) \sigma(s) /$ $\chi_{c l}(s)$, poles to the right of the imaginary axis cannot occur by admissibility (see the definition of admissibility earlier in this section). We can make sure that $T / H$ has no poles on the imaginary axis by letting $W$ have poles at the plant zeros on the imaginary axis, with multiplicities equal to those of the zeros.

Robustness: From recent work [1]-[7] it has become clear that in questions of robustness, in particular stability robustness, both the sensitivity function and the complementary sensitivity function of the closed-loop system play an important role. We shall show here, in a variation on previous arguments and in a somewhat exemplary fashion, that $S$ and $T$ may simultaneously determine stability robustness. To this end, we assume that the plant transfer function is nominally $H(s)=\psi(s) / \phi(s)$, and that the nominal closed-loop system is asymptotically stable. Next we assume that the plant transfer function is perturbed to

$$
H(s)=\frac{\psi^{\prime}(s)}{\phi^{\prime}(s)} h(s)
$$

where $\psi^{\prime}$ is a polynomial of the same degree as $\psi, \phi^{\prime}$ a polynomial of the same degree as $\phi$, and where $h(s):=\bar{\psi}(s) / \bar{\phi}(s)$ 
is the parasitic (or singular) part of the perturbation. We assume $\bar{\psi}$ and $\phi$ to be polynomial; the approach can be generalized to nonrational perturbations as well, however. We impose no restrictions on the relation of the roots of $\psi^{\prime}$ to those of $\psi$ or on the roots of $\phi^{\prime}$ to those of $\phi$, but we require the roots of $\bar{\phi}$ to be in the open left-half complex plane. It is clear that (2.1) represents a large although not exhaustive class of perturbations. It includes both structured perturbations (induced by parameter variations), represented by $\psi^{\prime}$ and $\phi^{\prime}$, and unstructured perturbations (induced by imperfect modeling), represented by $h$.

In the following the prime denotes the perturbed closed-loop system. With this convention we have $\chi_{c l}=\phi \sigma+\psi \rho, \chi_{c l}^{\prime}=$ $\phi^{\prime} \bar{\phi} \sigma+\psi^{\prime} \bar{\psi} \rho$ and it is easily established that

$$
\frac{\chi_{c}^{\prime} \prime}{\chi_{c} \phi}=\frac{\phi^{\prime}}{\phi} S+\frac{\psi^{\prime}}{\psi} h T \text {. }
$$

By the principle of the argument [17] it is easily shown that under the assumption that $\chi_{c l}$ and $\vec{\phi}$ both have all their roots in the open left-half plane, $\chi_{c l}^{\prime}$ has all its roots in the open left-half plane if and only if the image of $\chi_{c l}^{\prime}(i \omega) /\left(\chi_{c l}(i \omega) \bar{\phi}(i \omega)\right)$ does not encircle the origin as $\omega$ runs from $-\infty$ to $+\infty$. This means that the closedloop system remains stable under perturbation as long as this condition is satisfied. Equivalently, the closed-loop system remains stable under perturbation as long as

$$
\begin{aligned}
& \frac{\chi_{c l}^{\prime}(i \omega)}{\chi_{c l}(i \omega) \bar{\phi}(i \omega)}-1 \\
& \quad=\left(\frac{\phi^{\prime}(i \omega)}{\phi(i \omega)}-1\right) S(i \omega)+\left(\frac{\psi^{\prime}(i \omega)}{\psi(i \omega)} h(i \omega)-1\right) T(i \omega)
\end{aligned}
$$

does not encircle the point -1 .

A sufficient condition for the right-hand side of (2.3) not to encircle the point -1 is that its modulus is less than 1 for all $\omega$. Suppose that there exist rational functions $V_{1}, W_{1}$, and $W_{2}$ such that the various relative perturbations can be bounded as follows:

$$
\begin{gathered}
\left|\frac{\phi^{\prime}(i \omega)}{\phi(i \omega)}-1\right| \leq\left|V_{1}(i \omega)\right|,\left|\frac{\psi^{\prime}(i \omega)}{\psi(i \omega)}-1\right| \leq\left|W_{1}(i \omega)\right|, \\
|h(i \omega)-1| \leq\left|W_{2}(i \omega)\right|
\end{gathered}
$$

for all $\omega$. Then since

$$
\frac{\psi^{\prime}}{\psi} h-1=\left(\frac{\psi^{\prime}}{\psi}-1\right)(h-1)+\left(\frac{\psi^{\prime}}{\psi}-1\right)+(h-1),
$$

twofold application of the inequality

$$
\left|\sum_{i=1}^{n} x_{i}\right|^{2} \leq n \sum_{i=1}^{n}\left|x_{i}\right|^{2}
$$

shows that the right-hand side of (2.3) may be bounded from above by $2\left(\left|V_{1} S\right|^{2}+3\left(\left|W_{1} W_{2}\right|^{2}+\left|W_{1}\right|^{2}+\left|W_{2}\right|^{2}\right)|T|^{2}\right)$, where the argument is $i \omega$ throughout. Thus, a sufficient condition for stability of the closed-loop system under perturbations satisfying (2.4) is that an expression of the form

$$
|V(i \omega) S(i \omega)|^{2}+|W(i \omega) T(i \omega)|^{2}
$$

is bounded by $1,|V|^{2}:=2\left|V_{1}\right|^{2}$ and $|W|^{2}:=6\left(\left|W_{1} W_{2}\right|^{2}+\right.$ $\left.\left|W_{1}\right|^{2}+\left|W_{2}\right|^{2}\right)$. The point that we wish to make is that given information about the possible relative perturbations in the form (2.4), robust stability can be ensured by bounding an expression of the form (2.7), which is a weighted combination of $|S(i \omega)|^{2}$ and $|T(i \omega)|^{2}$.

Command Response: In single-degree-of-freedom control systems the control system transfer function from the command input to the controlled output equals the complementary sensitivity function $T$. The design requirement that stipulates the sensitivity function to be small over the frequency range of the disturbance by implication requires $T=1-S$ to be close to 1 over that same frequency range. This in turn ensures good response to command signals in that frequency range. It is assumed that any corrections to the desired response to command signals can be accomplished by a suitable prefilter in a two-degree-of-freedom control configuration, and we impose no additional requirements on the loop properties.

Minimax Optimization: The conclusion from the previous considerations is that various control system design objectives, such as disturbance attenuation and rejection, power and bandwidth limitation and robustness, can be expressed in the form of required bounds on $|V(i \omega) S(i \omega)|, \quad|W(i \omega) T(i \omega)|$ and $\left|V^{\prime}(i \omega) S(i \omega)\right|^{2}+\left|W^{\prime}(i \omega) T(i \omega)\right|^{2}$, with $V, W, V^{\prime}$, and $W^{\prime}$ suitable weighting functions. Equivalently, the design objectives can be expressed in terms of required bounds on $|V(i \omega) S(i \omega)|^{2}$, $|W(i \omega) T(i \omega)|^{2}$ and $\left|V^{\prime}(i \omega) S(i \omega)\right|^{2}+\left|W^{\prime}(i \omega) T(i \omega)\right|^{2}$. Since in each instance the smaller the bound is, the better the design objective is achieved, it is attractive to minimize the bounds in some way. We shall do this by minimizing the least upper bound of an expression of the form

$$
\begin{aligned}
& a|V(i \omega) S(i \omega)|^{2}+b|W(i \omega) T(i \omega)|^{2}+c\left[\left|V^{\prime}(i \omega) S(i \omega)\right|^{2}\right. \\
& \left.\quad+\left|W^{\prime}(i \omega) T(i \omega)\right|^{2}\right]
\end{aligned}
$$

where the nonnegative constants $a, b$, and $c$ reflect the importance of each term. Given an upper bound on (2.8), corresponding upper bounds on each of the terms can immediately be ascertained. Simplifying the notation by combining terms and renaming coefficients, we are thus led to the problem of minimizing an expression of the form

$$
\sup _{\omega}\left[|V(i \omega) S(i \omega)|^{2}+|W(i \omega) T(i \omega)|^{2}\right]
$$

with $V$ and $W$ suitable weighting functions. The solution of this problem is the subject of the next section.

\section{Solution of the Minimax Problem}

In this section we study the problem of minimizing the criterion (2.9).

Assumptions: We first introduce and explain a number of assumptions. To begin with, the weighting functions $V$ and $W$ are restricted to rational functions of the form

$$
V(s)=\frac{\alpha_{1}(s)}{\beta_{1}(s)}, W(s)=\frac{\alpha_{2}(s)}{\beta_{2}(s)}
$$

where $\alpha_{1}, \alpha_{2}, \beta_{1}$, and $\beta_{2}$ are real polynomials such that deg $\left(\alpha_{1}\right)$ $=: a_{1}, \operatorname{deg}\left(\alpha_{2}\right)=: a_{2}, \operatorname{deg}\left(\beta_{1}\right)=: b_{1}, \operatorname{deg}\left(\beta_{2}\right)=: b_{2}$, satisfying the following hypotheses.

a) $a_{1} \leq b_{1}$ and $a_{2}=b_{2}+e$, with $e$ a nonnegative integer.

b) $\alpha_{1}$ and $\alpha_{2}$ have all their roots in the closed left-half plane; $\beta_{1}$ and $\beta_{2}$ have all their roots in the closed left-half plane and have no common roots; $\alpha_{1}$ and $\beta_{1}$ have no common roots; $\alpha_{2}$ and $\beta_{2}$ have no common roots.

c) The polynomial $\gamma:=\alpha_{1} \alpha_{1}^{*} \beta_{2} \beta_{2}^{*}+\alpha_{2} \alpha_{2}^{*} \beta_{1} \beta_{1}^{*}$ has no roots on the imaginary axis.

Here if $f$ is any rational function, $f^{*}(s):=f(-s)$. Assumption a) ensures that $V(s)=0(1)$ and $W(s)=0\left(s^{e}\right)$ for $|s| \rightarrow \infty$, which is entirely acceptable in view of the discussion of the preceding section. The assumptions b) cause no loss of generality, except (very slightly) that $\beta_{1}$ and $\beta_{2}$ have no common roots. Assumption c) is needed to ensure that the problem has a solution at all.

Further to the assumptions at the end of Section I we introduce the factorizations

$$
\phi=\phi_{+} \phi_{o} \phi_{-}, \psi=\psi_{+} \psi_{o} \psi_{-}
$$


where $\phi_{+}, \phi_{o}, \phi_{-}, \psi_{+}, \psi_{o}$, and $\psi_{-}$are real polynomials with deg $\left(\phi_{+}\right)=: n_{+}, \operatorname{deg}\left(\phi_{0}\right)=: n_{o}, \operatorname{deg}\left(\phi_{-}\right)=: n_{-}, \operatorname{deg}\left(\psi_{+}\right)=:$ $m_{+}, \operatorname{deg}\left(\psi_{o}\right)=: m_{o}$ and $\operatorname{deg}\left(\psi_{-}\right)=: m_{-}$, such that $\phi_{+}$and $\psi_{+}$ have all their roots in the open right-half plane, $\phi_{o}$ and $\psi_{o}$ have all their roots on the imaginary axis, and $\phi_{-}$and $\psi_{-}$have all their roots in the open left-half plane.

Admissible Controllers: We restrict the admissible controllers to those that have a rational transfer function $G$ such that the closed-loop system has all its poles in the closed left-half plane. In the preceding section we have seen that if $W(s)=0\left(s^{e}\right)$ as $|s| \rightarrow$ $\infty$, with $e$ the plant pole excess, the controller transfer function is necessarily proper, provided (2.9) has a finite minimum at all. Later (Lemma 1) we shall see under what conditions on $V$ and $W$ the closed-loop system actually has all its poles in the open lefthalf plane.
Substitution of these formulas into (3.3) immediately shows that $Z(s)=\lambda^{2}$ is equivalent to

$$
\alpha_{1} \alpha_{1}^{*} \phi_{+} \phi_{+}^{*} \theta \theta^{*}+\alpha_{2} \alpha_{2}^{*} \psi_{+} \psi_{+}^{*} \zeta \zeta^{*}=\lambda^{2} \chi \chi^{*} .
$$

This equation constitutes a basic relation. Another crucial result follows by completing the square on the right-hand side of (3.3), and writing $Z$ in the form

$$
Z=\frac{\left(V V^{*} S-W W^{*} T\right)^{*}\left(V V^{*} S-W W^{*} T\right)+V V^{*} W W^{*}}{V V^{*}+W W^{*}}
$$

Substituting $V$ and $W$ as given by (3.1) and $S$ and $T$ as given by (3.9), it follows that

$$
Z=\frac{\left(\alpha_{1} \alpha_{1}^{*} \beta_{2}^{*} \phi_{+} \theta-\alpha_{2} \alpha_{2}^{*} \beta_{1}^{*} \psi_{+} \zeta\right)\left(\alpha_{1} \alpha_{1}^{*} \beta_{2}^{*} \phi_{+} \theta-\alpha_{2} \alpha_{2}^{*} \beta_{1}^{*} \psi_{+} \zeta\right)^{*}+\alpha_{1} \alpha_{1}^{*} \alpha \alpha_{2}^{*} \chi \chi^{*}}{\left(\beta_{1} \beta_{1}^{*} \alpha_{2} \alpha_{2}^{*}+\beta_{2} \beta_{2}^{*} \alpha_{1} \alpha_{1}^{*}\right) \chi \chi^{*}} .
$$

Preliminaries: In this subsection we introduce the equations that will determine the solution of the minimax problem. First, we define the rational function

$$
Z(s):=V(s) V(-s) S(s) S(-s)+W(s) W(-s) T(s) T(-s) .
$$

Thus, our problem is that of minimizing $\sup _{\omega} Z(i \omega)$. It will turn out that control systems that achieve this minimum have the property that

$$
Z(s)=\lambda^{2}
$$

with $\lambda$ a real constant, and we shall first consider which controllers from the admissible class achieve this. Suppose that the controller transfer function is $G=\rho / \sigma$, with $\rho$ and $\sigma$ real polynomials. Then

$$
L=H G=\frac{\psi_{+} \psi_{o} \psi_{-} \rho}{\phi_{+} \phi_{o} \phi_{-} \sigma}
$$

Without loss of generality we can choose $\rho$ and $\sigma$ such that all factors in the numerator and denominator of $L$ that have their roots in the closed left-half plane cancel. We, therefore, let $\rho$ have a factor $\phi_{-} \phi_{o}$ and let $\sigma$ have a factor $\psi_{-} \psi_{o}$. On the other hand, from (1.6) and (3.1)

$$
V S=\frac{\alpha_{1} \phi \sigma}{\beta_{1} \chi_{c l}}, W T=\frac{\alpha_{2} \psi \rho}{\beta_{2} \chi_{c l}} .
$$

Since we want $Z(s)=\lambda^{2}, V V^{*} S S^{*}$ cannot have poles at the roots of $\beta_{\mathrm{I}} \beta_{1}^{*}$ and $W W^{*} T T^{*}$ cannot have poles at the roots of $\beta_{2} \beta_{2}^{*}$. These requirements are satisfied when $\sigma$ has a factor $\beta_{1}$ and $\rho$ has a factor $\beta_{2}$.

In view of these observations, we let $\sigma=\psi_{-} \psi_{o} \beta_{1} \theta$ and $\rho=$ $\phi_{-} \phi_{o} \beta_{2} \zeta$, with $\theta$ and $\zeta$ real polynomials to be determined. This means that the controller transfer function is chosen as

$$
G=\frac{\phi_{-} \phi_{o} \beta_{2} \zeta}{\psi_{-} \psi_{o} \beta_{1} \theta}
$$

With this controller, the closed-loop characteristic polynomial is $\chi_{c l}=\phi_{-} \phi_{o} \psi_{-} \psi_{o} \chi$, where the polynomial $\chi$ is defined by

$$
\chi:=\beta_{1} \phi_{+} \theta+\beta_{2} \psi_{+} \zeta
$$

Correspondingly, the sensitivity function $S$ and its complement $T$ are given by

$$
S=\frac{\beta_{1} \phi_{+} \theta}{\chi}, T=\frac{\beta_{2} \psi_{+} \zeta}{\chi}
$$

Therefore, $Z(s)=\lambda^{2}$ iff

$$
\begin{aligned}
& \left(\alpha_{1} \alpha_{1}^{*} \beta_{2}^{*} \phi_{+} \theta-\alpha_{2} \alpha_{2}^{*} \beta_{1}^{*} \psi_{+} \zeta\right)\left(\alpha_{1} \alpha_{1}^{*} \beta_{2}^{*} \phi_{+} \theta-\alpha_{2} \alpha_{2}^{*} \beta_{1}^{*} \psi_{+} \zeta\right)^{*} \\
& =\lambda^{2}\left(\gamma-\frac{1}{\lambda^{2}} \eta\right) \chi \chi^{*}
\end{aligned}
$$

where

$$
\gamma:=\beta_{1} \beta_{1}^{*} \alpha_{2} \alpha_{2}^{*}+\beta_{2} \beta_{2}^{*} \alpha_{1} \alpha_{1}^{*}, \eta:=\alpha_{1} \alpha_{1}^{*} \alpha_{2} \alpha_{2}^{*} .
$$

We now define the polynomial $\pi_{\lambda}$, for those real $\lambda$ for which it exists, as the real polynomial of degree $b_{1}+b_{2}+e$ with nonnegative coefficients and all its roots in the closed left-half plane, such that

$$
\pi_{\lambda} \pi_{\lambda}^{*}=\gamma-\frac{1}{\lambda^{2}} \eta
$$

The polynomial $\pi_{\lambda}$ is well-defined for $|\lambda| \geq \lambda_{o}$, where $\lambda_{o} \geq 0$ is the first value of $\lambda$ for which the right-hand side of (3.15) either loses degree or assumes a root on the imaginary axis as $|\lambda|$ is decreased from $\infty$. By assumption c), $\lambda_{o}<\infty$. Returning to (3.13), we observe that there are many ways of factoring this equation. It will turn out that the factorization we need is

$$
\alpha_{1} \alpha_{1}^{*} \beta_{2}^{*} \phi_{+} \theta-\alpha_{2} \alpha_{2}^{*} \beta_{1}^{*} \psi+\zeta=\lambda \pi_{\lambda} \chi^{*} .
$$

Before proceeding, we summarize some properties of the controller (3.7).

Lemma 1: Consider the controller (3.7), and suppose that the polynomials $\theta$ and $\zeta$ satisfy the equation (3.16), with $\chi$ given by (3.8), for some real $\lambda$. Then the following holds. a) $Z(s)=\lambda^{2}$. b) If the polynomial $\chi$ has all its roots in the open left-half plane, the closed-loop system has all its poles in the closed left-half plane. c) If, in addition, $\beta_{1}$ has a factor $\phi_{o}$ and $\beta_{2}$ has a factor $\psi_{o}$, and these factors are canceled in the controller transfer function (3.7), the closed-loop system has all its closed-loop poles in the open lefthalf plane.

Proof: a) That $Z(s)=\lambda^{2}$ follows by substituting (3.16) into (3.12). b) As we have seen following (3.7), the closed-loop polynomial is $\phi_{-} \phi_{o} \psi_{-} \psi_{o} \chi$, so that under the hypothesis the closed-loop poles are all in the closed left-half plane. c) If in the controller transfer function the factors $\phi_{o}$ and $\psi_{o}$ are canceled, the closed-loop characteristic polynomial is $\phi_{-} \psi_{-} \chi$, which under the hypothesis has all its roots in the open left-half plane.

The final step in this subsection is to establish the existence of a solution to (3.16). To this end, we first define the integers

$$
x:=n_{+}+m_{+}+b_{1}+b_{2}+e, t:=m_{+}+b_{2}+e, z:=n_{+}+b_{1} .
$$


Theorem 1: a) For $|\lambda| \geq \lambda_{o}$ the polynomial equation (3.16), with $\chi$ defined by (3.8), has a family of solutions $\chi_{\lambda}, \theta_{\lambda}, \zeta_{\lambda}$ with $\operatorname{deg}\left(\chi_{\lambda}\right) \leq x, \operatorname{deg}\left(\theta_{\lambda}\right) \leq t, \operatorname{deg}\left(\zeta_{\lambda}\right) \leq z$, unique within multiplication by a continuous function of $\lambda$, whose coefficients are continuous functions of $\lambda$, such that for $|\lambda|$ sufficiently large the polynomial $\chi_{\lambda}$ has degree $x$ and has all its roots in the open left-half complex plane. b) Suppose that $\chi_{\lambda_{o}}$ or $\chi_{-\lambda_{o}}$ has a root in the open right-half plane. Then there exists a $\lambda$ with $|\lambda|>\lambda_{0}$ such that $\operatorname{deg}\left(\chi_{\lambda}\right) \leq x-1, \operatorname{deg}\left(\theta_{\lambda}\right) \leq t-1, \operatorname{deg}\left(\zeta_{\lambda}\right) \leq z-1$ and $\chi_{\lambda}$ has all its roots in the open left-half plane.

In part b) of the theorem, it is to be understood that if a polynomial is assigned the degree -1 , it is the zero polynomial. The proof of the theorem is given in Appendix A. That of part a) relies on first proving that (3.10) has a suitable solution (by the implicit function theorem), and then showing that this solution also solves (3.16). Part b) follows from the fact that under the hypothesis at least one root of $\chi_{\lambda}$ crosses over from the left- to the right-half plane for some $|\lambda|>\lambda_{o}$; this root can be canceled, resulting in a solution of reduced degree.

Solution of the Minimax Problem: Before presenting the details of the solution of the minimax problem, we establish an important property of the number $\lambda_{o}$ previously defined.

Lemma 2: For any controller, $\sup _{\omega} Z(i \omega) \geq \lambda_{o}^{2}$.

Proof: From (3.12) it is seen that $Z(i \omega) \geq \eta(i \omega) / \gamma(i \omega)$ for all real $\omega$. It follows that $\sup _{\omega} Z(i \omega) \geq \sup _{\omega}[\eta(i \omega) / \gamma(i \omega)]=$ inf $\left\{\lambda^{2}: \eta(i \omega) / \gamma(i \omega) \leq \lambda^{2}\right.$ for all $\left.\omega\right\}=\inf \left\{\lambda^{2}: \gamma(i \omega)-\eta(i \omega) / \lambda^{2} \geq 0\right.$ for all $\omega\}=\lambda_{o}^{2}$. The last step is detailed in Appendix $B$.

The following describes the solution to the problem of minimizing (2.9) in a somewhat exceptional situation.

Theorem 2: Suppose that $\chi_{\lambda_{0}}$ or $\chi_{-\lambda_{o}}$ has all its roots in the closed left-half plane. Then the controller (3.7), with $\theta$ replaced with $\theta_{\lambda_{o}}$, respectively $\theta_{-\lambda_{o}}$, and $\zeta$ replaced with $\zeta_{\lambda_{o}}$, respectively, $\zeta_{-\lambda_{o}}$, minimizes (2.9), and the minimal value is $\lambda_{o}^{2}$.

Proof: Any roots of $\chi_{\lambda_{o}}$, respectively $\chi_{-\lambda_{o}}$, on the imaginary axis may be canceled as in the proof of Theorem $1 \mathrm{lb}$ ). As the remaining roots are in the open left-half plane, by Lemma 1 the closed-loop system has all its poles in the closed left-half plane and achieves $Z(i \omega)=\lambda_{o}^{2}$. Then by Lemma 2 the control system minimizes (2.9).

We briefly return to this situation in the Corollary to Theorem 3 . In case Theorem 2 does not apply, we introduce the auxiliary problem of minimizing

$$
\int_{-\infty}^{\infty} Z(i \omega) \Sigma(i \omega) d \omega
$$

where $\Sigma(s)$ is a rational, strictly proper function of $s$ with real coefficients such that $\Sigma(i \omega)$ is real and positive for $-\infty<\omega<$ $\infty$. The following result explains our interest in controllers such that $Z(i \omega)=$ constant.

Lemma 3: Suppose that (for a given $\Sigma$ ) the auxiliary criterion (3.18) is minimized by an admissible controller that makes $Z(i \omega)$ a constant $\lambda^{2}$. Then this controller also minimizes $\sup _{\omega} Z(i \omega)$.

Proof: This result is well known from statistical decision theory (see, e.g., [18]) and its proof is very simple. Suppose that there exists an admissible controller leading to a function $\tilde{Z}$ such that $\sup _{\omega} \tilde{Z}(i \omega)<\lambda^{2}$. Then necessarily $\tilde{Z}(i \omega)<\lambda^{2}=Z(i \omega)$ for all $\omega$. As a result, $\int_{-\infty}^{\infty} \tilde{Z}(i \omega) \Sigma(i \omega) d \omega<\int_{-\infty}^{\infty} Z(i \omega) \Sigma(i \omega) d \omega$, which contradicts the assumption that $Z$ minimizes (3.18).

A sufficient condition for the minimization of $(3.18)$ is the following.

Lemma 4: A sufficient condition for the minimization of (3.18) is that $\Sigma \phi \psi\left(V V^{*} S^{*}-W W^{*} T^{*}\right)$ has all its poles in the closed left-half plane. If a minimizing solution exists, it is unique.

A proof of this lemma is given in Appendix C. We are now able to state the solution to the problem of minimizing (2.9) in case Theorem 2 does not apply.

Theorem 3: Suppose that $\chi_{\lambda_{0}}$ or $\chi_{-\lambda_{n}}$ has at least one root in the open right-half plane. According to Theorem lb) there exists a $\lambda$ with $|\lambda|>\lambda_{o}$ such that $\operatorname{deg}\left(\chi_{\lambda}\right) \leq x-1, \operatorname{deg}\left(\theta_{\lambda}\right) \leq t-1$, $\operatorname{deg}\left(\zeta_{\lambda}\right) \leq z-1$ and $\chi_{\lambda}$ has all its roots in the open left-half plane. Then the controller (3.7), with $\theta$ replaced with $\theta_{\lambda}$ and $\zeta$ replaced with $\zeta_{\lambda}$, uniquely minimizes (2.9), and the minimal value is $\lambda^{2}$.

Proof: We shall show that the solution $\chi_{\lambda}, \theta_{\lambda}, \zeta_{\lambda}$ of reduced degree minimizes an auxiliary criterion of the form (3.18), with $\Sigma$ suitably chosen. With $S$ and $T$ given by (3.9), the optimality condition of Lemma 4 takes the form that the rational function

$$
\Sigma \phi \psi \frac{\alpha_{1} \alpha_{1}^{*} \beta_{2} \phi_{+}^{*} \theta_{\lambda}^{*}-\alpha_{2} \alpha_{2}^{*} \beta_{1} \psi_{+}^{*} \zeta_{\lambda}^{*}}{\beta_{1} \beta_{2} \chi_{\lambda}^{*}}
$$

should have all its poles in the closed left-half plane. For controllers that satisfy $(3.16)$, this condition reduces to the requirement that

$$
\Sigma \phi \psi \frac{\lambda \pi_{\lambda}^{*} \chi_{\lambda}}{\beta_{1} \beta_{2} \chi_{\lambda}^{*}}
$$

have all its poles in the closed left-half plane. This requirement is indeed satisfied when

$$
\Sigma=\frac{\chi_{\lambda} \chi_{\lambda}^{*}}{\phi_{+} \phi_{+}^{*} \psi_{+} \psi_{+}^{*} \pi_{\lambda} \pi_{\lambda}^{*}} .
$$

Since for the particular solution of (3.16) that we are considering $\operatorname{deg}\left(\chi_{\lambda}\right) \leq x-1$, and $\operatorname{deg}\left(\pi_{\lambda}\right)=b_{1}+b_{2}+e$ for $|\lambda|>\lambda_{o}, \Sigma$ as given is strictly proper as required. Thus, by Lemma 4 , the controller we consider minimizes the auxiliary criterion (3.18). Since by Lemma 1 for this controller $Z(i \omega)=\lambda^{2}=$ constant, it follows from Lemma 3 that this controller minimizes (2.9). It remains to prove uniqueness. Suppose first that there exists a controller resulting in a $Z$ such that $\sup _{\omega} Z(i \omega)=\lambda^{2}$ but $Z(i \omega)<$ $\lambda^{2}$ over some nonzero interval. Then since $\Sigma$ is strictly positive, this controller would achieve a smaller value for the auxiliary criterion (3.18), with $\Sigma$ given by (3.21), than the controller considered so far. This contradicts the fact that the latter controller minimizes the auxiliary criterion. We conclude that for any controller that minimizes $(2.9)$ necessarily $Z(i \omega)=\lambda^{2}$. Therefore, such a controller minimizes the auxiliary criterion (3.18) with $\Sigma$ given by (3.21). Since by Lemma 4 the controller that minimizes the auxiliary criterion is uniquely determined, also the minimax controller is uniquely determined.

Theorems 2 and 3 show that the solution of the minimax optimization problem may be reduced to determining suitable solutions to the polynomial equation (3.16), with $\chi$ defined by (3.8). Since solutions to (3.16) also satisfy (3.10), alternatively one may solve (3.10), with $\chi$ given by (3.8), but only solutions qualify that satisfy (3.16). For a further discussion see Section IV and Example 2 in Section VI.

Corollary: $\chi_{\lambda o}$ has all its roots in the closed left-half plane if and only if $\chi_{-\lambda o}$ has all its roots in the closed left-half plane.

Proof: Let $\chi_{\lambda o}$ have all its roots in the closed left-half plane, but suppose that $x-\lambda_{0}$ has a root in the open right-half plane. Then by Theorem 2 there exists an optimal controller that achieves $\sup _{\omega} Z(i \omega)=\lambda_{o}^{2}$, while by Theorem 3 there exists an optimal controller that achieves $\sup _{\omega} Z(i \omega)=\lambda^{2}>\lambda_{o}^{2}$. This is a contradiction. The proof when $\chi-\lambda_{o}$ has all its roots in the closed left-half plane is similar.

In case Theorem 2 applies (which is a somewhat exceptional situation) the optimal controller may be nonunique. An example is given in Section VI (Example 1).

We conclude this section with a remark about the order of the optimal controller. Suppose that i) $e=n-m$, and ii) all roots of $\beta_{1}$ are also roots of $\phi_{o} \phi_{-}$and all roots of $\beta_{2}$ are also roots of $\psi_{0} \psi_{-}$. Then it is easy to check that if Theorem 2 applies, the controller order is $n$, while in case Theorem 3 applies it is $n-1$. Here $n$ is the plant order. Assumption i) ensures that the controller is proper, while assumption ii) includes the situation where $\phi_{o}$ is a factor of $\beta_{1}$ and $\psi_{o}$ is a factor of $\beta_{2}$, which guarantees the closed- 
loop system to be asymptotically stable. If i) and ii) do not hold, the controller order may be less or greater than $n$, respectively, $n$ - 1. A controller order less than $n$, respectively, $n-1$ is achieved only if the requirement that the controller be proper is dropped.

\section{NumERICAL SOLUTION}

Unless the various polynomials that are involved are of very low degree, it is not feasible to solve the polynomial equations that determine the solution of the minimax problem in closed form. Often it will be necessary to take recourse to numerical computation.

To check whether Theorem 2 applies, it is necessary to solve the (overdetermined) linear equation (3.16), with $\chi$ defined by (3.8), for $\lambda= \pm \lambda_{o}$. This is a routine problem from numerical linear algebra. Algorithms for the factorization (3.15) are well known (see, e.g., Kucera [19]).

If for $\lambda= \pm \lambda_{o}$ no solution is obtained such that $\chi$ has all its roots in the closed left-half plane it is useful to solve the equation (3.16) for a number of trial values of $\lambda$ in order to obtain an estimate of the solution for which the first root of $\chi$ crosses over to the right-half plane as $|\lambda|$ is decreased from $\infty$.

To find the exact solution of reduced degree, it seems most convenient to solve $\chi, \theta, \xi$, and $\lambda$, with $\operatorname{deg}(\chi)=x-1, \operatorname{deg}(\theta)$ $=t-1, \operatorname{deg}(\zeta)=z-1$, from (3.10), with $\chi$ given by (3.8). Since these equations are homogeneous, it is necessary to add a normalization condition, for instance that $\chi$ is monic. This results in a set of nonlinear equations with as many unknowns as equations, which may be solved by a Newton-Raphson approach, using the approximate solution obtained as indicated in the preceding paragraph as a starting solution. Since solutions to (3.10), even if $\chi$ has all its roots in the open left-half plane, do not necessarily satisfy (3.16), the latter equation always has to be checked (see Example 2 in Section VI).

Computer codes (in Pascal) that implement these algorithms are available [20].

\section{Specialization to the Case $W=0$}

In this section we consider the specialization of the minimax problem to the case where $W=0$, which is the problem solved by Zames and Francis [7], [8]. To this end we take $\alpha_{2}=0, \beta_{2}=1$. It immediately follows that $\gamma=\alpha_{1} \alpha_{1}^{*}, \eta=0, \lambda_{o}=0$, and $\pi_{\lambda}=$ $\alpha_{1}$, so that (3.8) and (3.16) reduce to $\chi=\beta_{1} \phi_{+} \theta+\psi_{+} \zeta, \alpha_{1}^{*} \phi_{+} \theta$ $=\lambda \chi^{*}$. Elimination of $\chi$ leads to the equation

$$
\frac{1}{\lambda} \alpha_{1} \phi_{+}^{*} \theta^{*}=\beta_{1} \phi_{+} \theta+\psi+\zeta
$$

where $\operatorname{deg}(\theta)=t=m_{+}, \operatorname{deg}(\zeta)=z=n_{+}+b_{1}$. Note that although this equation does not appear in the work of Zames and Francis, it can immediately be obtained from their results.

We first check whether Theorem 2 applies. For $\lambda=\lambda_{o}=0$ the equation (5.1) has the solution $\hat{\theta}=\psi_{+}^{*}, \zeta=\alpha_{1} \phi_{+}^{*}, \chi=\alpha_{1} \phi_{+}^{*} \psi_{+}$, where $\hat{\theta}:=\theta / \lambda$. The polynomial $\chi$ has its roots in the open lefthalf plane if and only if $\psi_{+}=$constant (note that by assumption $\mathrm{c}$ ) $\alpha_{1}$ has all its poles in the open left-half plane). This solution corresponds to the well-known fact [8] that if the plant has no right-half plane zeros, one can make $|S(i \omega)|$ uniformly arbitrarily close to 0 by making the gain large enough.

When the plant possesses right-half plane zeros, Theorem 3 applies and we need to solve (5.1) with deg $(\theta)=m_{+}-1$, deg $(\zeta)=n_{+}+b_{1}-1$. If $n_{+}+b_{1}=0$, by assumption a) $a_{1}=0$, and (5.1) has the trivial solution $\lambda=\alpha_{1} / \beta_{1}, \theta=1, \zeta=0$. In all other cases, (5.1) can be reduced to a (generalized) eigenvalue problem as follows. Define $\theta$ and $\zeta$ as the $m_{+-}$, respectively, $\left(n_{+}\right.$ $+b_{1}$ )-dimensional column vectors whose elements are the coefficients of the polynomial $\theta$, respectively, $\zeta$, arranged in order of increasing power. Then in matrix form the polynomial equation
(5.1) can be written as

$$
\begin{aligned}
& \frac{1}{\lambda} T_{n_{+}+m_{+}+b_{1}, m_{+}}\left(\alpha_{1} \phi_{+}^{*}\right) J_{m_{+}} \theta \\
& \quad=T_{n_{+}+m_{+}+b_{1}, m_{+}}\left(\beta_{1} \phi_{+}\right) \theta+T_{n_{+}+m_{+}+b_{1}, n_{+}+b_{1}}\left(\psi_{+}\right) \zeta .
\end{aligned}
$$

Here if $\alpha$ is any polynomial, $T_{n, m}(\alpha)$ is the $n \times m$ lower triangular Toeplit $z$ matrix formed from the coefficients of $\alpha$ (see Kailath [21]); furthermore, $J_{n}$ is the $n \times n$ diagonal matrix $J_{n}$ $:=\operatorname{diag}(1,-1,1,-1, \cdots)$. The equation $(5.2)$ can be rearranged as

$$
\left(\frac{1}{\lambda} A-B\right)\left[\begin{array}{l}
\theta \\
\zeta
\end{array}\right]=0
$$

where $A$ and $B$ are the $\left(n_{+}+m_{+}+b_{1}\right) \times\left(n_{+}+m_{+}+b_{1}\right)$ matrices

$$
A:=\left(T\left(\alpha_{1} \phi_{+}^{*}\right) J, 0\right), B:=\left(T\left(\beta_{1} \phi_{+}\right), T\left(\psi_{+}\right)\right) .
$$

Here for brevity we omitted the indexes. By the assumptions on $\beta_{1}, \phi_{+}$, and $\psi_{+}$the matrix $B$ is nonsingular; hence (5.3) is equivalent to $(R / \lambda-I) \operatorname{col}(\theta, \zeta)=0$, where $R:=B^{-1} A$. It is seen that $R$ is of the form

$$
\left[\begin{array}{ll}
R_{11} & 0 \\
R_{21} & 0
\end{array}\right]
$$

where $R_{11}$ is $m_{+} \times m_{+}$and $R_{21}$ is $\left(n_{+}+b_{1}\right) \times m_{+}$. As a result, we have

$$
R_{11} \theta=\lambda \theta, \zeta=\frac{1}{\lambda} R_{21} \theta
$$

Clearly, $\lambda$ is an eigenvalue of $R_{11}$ and $\theta$ a corresponding eigenvector. Now, from the proof of Theorem $1 b$ ) it follows that if there are several values of $\lambda$ that yield a solution of (5.1) of reduced degree, the one we are looking for (namely that for which $\chi$ has all its roots in the open left-half plane) is that for which $\lambda$ is the largest (in absolute value). Thus, $\lambda$ is the largest (in absolute value) of the real eigenvalues of $R_{11}$.

The eigenvalue problem (5.6) that results from (5.1) may be routinely solved. This approach is offered as an alternative to the methods proposed by Zames and Francis [7], [8]. It leads of course to the same solutions. An example is given in Section VI (Example 3).

\section{EXAMPLES}

Example 1: The first example serves to illustrate that there are cases where the lower bound $\lambda_{o}^{2}$ is achieved, and that in these cases the solution may be nonunique. Take $H=1, V=(s+\epsilon) /$ $(s+1), W=s$, where $0<\epsilon<1$. Then the polynomial $\pi_{\lambda}$ is defined by $\pi_{\lambda} \pi_{\lambda}^{*}=\left(1-1 / \lambda^{2}\right) s^{4}+\left(-2+\epsilon^{2} / \lambda^{2}\right) s^{2}+\epsilon^{2}$. From this it may be verified that $\lambda_{o}=1$ and $\pi_{\lambda_{0}}=\left(2-\epsilon^{2}\right)^{1 / 2} s+\epsilon$. For $\lambda= \pm 1$ the equations (3.8) and (3.16) reduce to $\chi=(s+$ 1) $\theta+\zeta, \quad \pm\left(\left(2-\epsilon^{2}\right)^{1 / 2} s+\epsilon\right) \chi^{*}=\left(-s^{2}+\epsilon^{2}\right) \theta+s^{2}(-s+1) \zeta$, where $\operatorname{deg}(\chi)=x=2, \operatorname{deg}(\theta)=t=1$ and $\operatorname{deg}(\zeta)=z=1$. It is straightforward to solve the linear equations that result from expanding the polynomial equations in terms of their coefficients. It is found that for $\lambda=1$ the solution may be written as $\theta=(\epsilon+$ 1) $+\left(\left(2-\epsilon^{2}\right)^{1 / 2}-1\right) s, \zeta=\left(\epsilon^{2}-1\right), \chi=\epsilon(1+\epsilon)+(\epsilon+(2$ $\left.\left.-\epsilon^{2}\right)^{1 / 2}\right) s+\left(\left(2-\epsilon^{2}\right)^{1 / 2}-1\right) s^{2}$, while for $\lambda=-1$ the solution is $\theta=(\epsilon-1)+\left(\left(2-\epsilon^{2}\right)^{1 / 2}+1\right) s, \zeta=\left(1-\epsilon^{2}\right), \chi=\epsilon(1-\epsilon)$ $+\left(\epsilon+\left(2-\epsilon^{2}\right)^{1 / 2}\right) s+\left(\left(2-\epsilon^{2}\right)^{1 / 2}+1\right) s^{2}$. For $0<\epsilon<1$ in both cases $\chi$ has all its roots in the open left-half plane, and hence we have here two distinct asymptotically stable control systems that both achieve $Z(i \omega)=\lambda_{o}^{2}$.

Example 2: This example shows that solving (3.10) may yield nonoptimal solutions even if $\chi$ has all its roots in the open left-half 
plane. Let $H=1, V=1 /(1+\tau s), W=s$. Then the equations (3.8) and (3.10) take the form

$$
\chi=(1+\tau s) \theta+\zeta, \lambda^{2} \chi \chi^{*}=\theta \theta^{*}-s^{2} \zeta \zeta^{*} .
$$

For a solution of reduced degree, $\operatorname{deg}(\chi)=1, \operatorname{deg}(\theta)=0$, and $\operatorname{deg}(\zeta)=0$. Letting $\chi:=s+\chi_{0}, \theta=\theta_{0}, \zeta=\zeta_{0}$, with $\chi_{0}, \theta_{0}$, and $\zeta_{0}$ constants, it is not difficult to set up and solve the equations that follow from (6.1). For $\chi_{0}$ four solutions are found, namely

$$
\text { a) } \chi_{0}=\frac{1}{2 \tau}(1 \pm \sqrt{1+4 \tau}), \quad \text { b) } \chi_{0}=\frac{1}{2 \tau}(1 \pm \sqrt{1-4 \tau}) \text {. }
$$

In case a), for $\chi$ to have its roots in the left-half plane we need to replace the \pm sign with + . In case $b$ ), to obtain real solutions we need $4 \tau \leq 1$; if this is satisfied, $\chi$ has its roots in the left-half plane for both solutions. The corresponding values of $\lambda$ are (for the cases where $\chi$ has left-half plane roots only)

$$
\text { a) } \lambda^{2}=\frac{1}{4 \tau^{2}}(-1+\sqrt{1+4 \tau})^{2} \text {, b) } \lambda^{2}=\frac{1}{4 \tau^{2}}(-1 \pm \sqrt{1-4 \tau})^{2} \text {. }
$$

It can be verified that the values of $\lambda^{2}$ in case b) are always larger than that in case a); hence case b) cannot be optimal. Indeed, it turns out that the solution b) does not satisfy (3.16).

Example 3: To illustrate our results for the special case where $W=0$, we consider an example also solved by Francis and Zames [8]. Let $H(s)=(s-1)^{2} /\left((s+1)^{2}(-s+2)\right), V(s)=(s$ $+1) /(10 s+1)$, so that $\psi_{+}=(s-1)^{2}, \phi_{+}=-s+2, \alpha_{1}=s$ +1 , and $\beta_{1}=10 s+1$. Using the approach outlined in Section $\mathrm{V}$, it is easy to determine that the matrices $A$ and $B$ are given by

$$
A=\left[\begin{array}{rrrr}
2 & 0 & 0 & 0 \\
3 & -2 & 0 & 0 \\
1 & -3 & 0 & 0 \\
0 & -1 & 0 & 0
\end{array}\right], B=\left[\begin{array}{rrrr}
2 & 0 & 1 & 0 \\
19 & 2 & -2 & 1 \\
-10 & 19 & 1 & -2 \\
0 & -10 & 0 & 1
\end{array}\right]
$$

As a result,

$$
\begin{aligned}
& R_{11}=\left[\begin{array}{cc}
5 / 121 & 61 / 121 \\
61 / 121 & -127 / 121
\end{array}\right], \\
& R_{21}=\left[\begin{array}{cc}
232 / 121 & -122 / 121 \\
610 / 121 & -1391 / 121
\end{array}\right] .
\end{aligned}
$$

It is easily found that $R_{11}$ has the eigenvalues -1.24688 and 0.238613 . It follows that $\lambda=-1.24688$. The corresponding eigenvector of $R_{11}$ is $\theta=\operatorname{col}(-0.391346,1)$; hence $\theta=-$ $0.391346+$ s. From $\zeta=-1 / \lambda R_{21} \theta$ we find that $\zeta=1.41041$ $+10.8020 \mathrm{~s}$. Given $\lambda, \theta$, and $\zeta$, the controller transfer function $G$ and the resulting sensitivity function $S$ are easily found.

Example 4-Double Integrator: As a final example we take the feedback control of a double integrator, with the purpose of illustrating the practical value of the optimization technique discussed in this paper. We assume that the (nominal) plant transfer function is given by

$$
H(s)=k_{o} / s^{2}
$$

with $k_{o}$ a constant. We could take this as a model of the transfer function of a d.c. motor. We first discuss the various considerations in the choice of the weighting functions.

Disturbance Attenuation and Rejection: The plant has a double pole at the origin. Therefore, to achieve rejection of plantgenerated disturbances, $V$ should have a double pole at the origin. A preliminary choice is $V=1 /\left(\tau_{1} s\right)^{2}$, with the constant $\tau_{1}$ to be determined. This weighting function ensures rejection of constant disturbances and attenuation of very low-frequency disturbances.
To achieve disturbance attenuation over a wider frequency range, we modify $V V^{*}=1 /\left(\tau_{1} s\right)^{4}$ to

$$
V V^{*}=\frac{1}{\left(\tau_{1} s\right)^{4}}+1=\frac{1+\left(\tau_{1} s\right)^{4}}{\left(\tau_{1} s\right)^{4}} .
$$

Power and Bandwidth Limitation: Since $1 / H(s)=s^{2} / k_{o}$, we choose $W(s)=\left(\tau_{2} s\right)^{2}$, with the constant $\tau_{2}$ to be determined. This choice of $W$ makes sure that the controller transfer function will be proper.

Robustness: By way of illustration, suppose that the actual (perturbed) plant transfer function is

$$
H(s)=\frac{k}{s(s+\alpha)}
$$

where $k \neq k_{o}$ and $\alpha>0$. This perturbation might be caused by gain changes and the occurrence of friction in the motor. In the notation of Section II, we have $\psi=k_{o}, \phi=s^{2}, \psi^{\prime}=k, \phi^{\prime}=$ $s(s+\alpha), h=1$. Consequently,

$$
\frac{\psi^{\prime}-\psi}{\psi}=\frac{k-k_{o}}{k_{o}}, \frac{\phi^{\prime}-\phi}{\phi}=\frac{\alpha}{s}, h-1=0
$$

These relative perturbations can be bounded as in (2.4), where

$$
V_{1}=\epsilon, W_{1}=\frac{\alpha_{\max }}{s}, W_{2}=0
$$

Here $\epsilon$ is the maximal relative variation of the gain $k$, and $\alpha_{\max }$ the maximal value of $\alpha$. Pursuing the argument following (2.4), we see that robust stability is ensured when $|V S|^{2}+|W T|^{2}<1$ for all $\omega$, where

$$
V=\sqrt{2} \frac{\alpha_{\max }}{s}, W=\sqrt{2} \epsilon .
$$

Inspection of the weighting functions shows that for the inequality to be satisfied, $|S|$ should be (very) small at low frequencies and $|T|$ should be sufficiently less than $1 /(\epsilon \sqrt{2})$ at all frequencies. These requirements are fully compatible with the expected effects of the weighting furicions that were selected for disturbance attenuation and power and bandwidth limitation. Consequently, there is no need for modification of $V$ and $W$ for robustness improvement.

Solution of the Optimization Problem: We discuss the minimization of $\sup \left(|V S|^{2}+|W T|^{2}\right)$ for the plant $H(s)=k_{o}$ ' $s^{2}$, with

$$
V V^{*}=\frac{\left(1+\left(\tau_{1} s\right)^{4}\right.}{\left(\tau_{1} s\right)^{4}}, W=\left(\tau_{2} s\right)^{2}
$$

It may be checked that all necessary assumptions are satisfied. Next, it may be found that the defining equation (3.15) for the polynomial $\pi_{\lambda}$ and the equations (3.8), (3.16), and (3.10) take the form

$$
\begin{gathered}
\pi_{\lambda} \pi_{\lambda}^{*}=\tau_{1}^{4} \tau_{2}^{4}\left(1-\frac{1}{\lambda^{2}}\right) s^{8}+\left(\tau_{1}^{4}-\frac{1}{\lambda^{2}} \tau_{2}^{4}\right) s^{4}+1, \\
\chi=\tau_{1}^{2} s^{2} \theta+\zeta \\
\lambda \pi_{\lambda} \chi^{*}=\left(1+\tau_{1}^{4} s^{4}\right) \theta-\tau_{1}^{2} \tau_{2}^{4} s^{6} \zeta, \\
\lambda^{2} \chi \chi^{*}=\left(1+\tau_{1}^{4} s^{4}\right) \theta \theta^{*}+\tau_{2}^{4} s^{4} \zeta \zeta^{*}
\end{gathered}
$$

where we took $\phi_{+}=\psi_{+}=\phi_{-}=\psi_{-}=1, \phi_{o}=s^{2}, \psi_{o}=k_{0}$. The equations can be rewritten in terms of a single parameter $p$ $:=\left(\tau_{2} / \tau_{1}\right)^{4}$ by the substitution $\hat{s}=s \tau_{1}$. Immediately omitting the 
circumflex, we obtain

$$
\begin{gathered}
\pi_{\lambda} \pi_{\lambda}^{*}=\left(1-\frac{1}{\lambda^{2}}\right) s^{8}+\left(1-\frac{p}{\lambda^{2}}\right) s^{4}+1, \\
\chi=s^{2} \theta+\zeta, \\
\lambda \pi_{\lambda} \chi^{*}=\left(1+s^{4}\right) \theta-p s^{6} \zeta, \\
\lambda^{2} \chi \chi^{*}=\left(1+s^{4}\right) \theta \theta^{*}+p s^{4} \zeta \zeta^{*}
\end{gathered}
$$

From root locus considerations it follows that the lowest value $\lambda_{o}$ of $\lambda$ for which the factorization (6.17) is possible is assumed when the leading coefficient of the right-hand side vanishes; hence $\lambda_{o}=$ 1. We conjecture, and indeed this is borne out by what follows, that the lower bound $\lambda_{o}$ cannot be achieved, and immediately start looking for a solution of reduced degree. In the present case, $x=$ $4, t=2, z=2$; hence, we seek a solution with $\operatorname{deg}(\chi)=3$, deg $(\theta)=1, \operatorname{deg}(\zeta)=1$.

We choose to solve the linear equation (6.18) together with the quadratic equation (6.20) for the polynomials $\chi:=\chi_{0}+\chi_{1} s+$ $\chi_{2} s^{2}+s^{3}, \theta:=\theta_{o}+\theta_{1} s$ and $\zeta:=\zeta_{0}+\zeta_{1} s$ and for $\lambda$. It is a simple matter to set up the equations for the coefficients and reasonably straightforward to find that $\chi_{0}=\left(\left(\lambda^{4}-\lambda^{2}-p\right) /\right.$ $\left.\left(2 p \lambda^{3}\right)\right)^{1 / 2}, \chi_{1}=\left(\left(\lambda^{2}-1\right) / p\right)^{1 / 2}, \chi_{2}=\lambda \chi_{0}, \theta_{0}=\lambda \chi_{0}, \theta_{1}=1, \zeta_{0}$ $=\chi_{0}, \zeta_{1}=\chi_{1}$, while $\lambda$ satisfies the equation

$$
\left(\lambda^{4}-\lambda^{2}-p\right)^{2}-4 p^{1 / 2} \lambda^{5}\left(\lambda^{2}-1\right)^{1 / 2}=0 .
$$

In the process of obtaining these equations we used the fact that the coefficients of $\chi$ should all be positive.

Given $p,(6.21)$ can be solved numerically to obtain $\lambda$, and, from this, the complete solution. In each case that was tried, it was found that there are two real values of $\lambda$ satisfying the equations; only one of these results in real polynomials $\chi, \theta$, and $\zeta$, however. Table I lists the numerical solutions for a few values of $p$. In Fig. 2 , amplitude plots are given of the resulting sensitivity function $S$ and complementary sensitivity function $T$. For $p$ small (i.e., $\tau_{2} \ll$ $\tau_{1}$ ), the criterion is dominated by the term with $S$, and the modulus of the sensitivity function more or less behaves as $1 /|V|$. As $p$ increases, the term with $T$ assumes more and more influence, pushing the bandwidth down and causing $|T|$ to fall off more steeply, at the cost of peaking of $|S|$ near the cutoff frequency. The controller transfer function in each case is

$$
G(s)=\frac{\zeta_{0}+\zeta_{1} s}{k_{0} \tau_{1}^{2}\left(\theta_{0}+\theta_{1} s\right)}
$$

(still in terms of the dimensionless frequency defined before). The controller thus is of a conventional lead-lag type.

It is instructive to obtain asymptotic formulas for $p$ small. For $p$ $\downarrow 0$, the desired root of $(6.21)$ behaves as $\lambda^{2}-1+2^{4 / 3} p^{1 / 3}$. Correspondingly, $\chi \sim s^{3}+2^{1 / 6} p^{-1 / 3} s^{2}+2^{2 / 3} p^{-1 / 3} s+$ $2^{1 / 6} p^{-1 / 3}, \theta \sim s+2^{1 / 6} p^{-1 / 3}, \zeta \sim 2^{2 / 3} p^{-1 / 3} s+2^{1 / 6} p^{-1 / 3}$. Asymptotically, the polynomial $\chi$, which in this case is precisely the closed-loop characteristic polynomial, has the roots $2^{1 / 6} p^{-1 / 3}$ and $1 / 2 \sqrt{2}(-1 \pm i)$. The latter pair of closed-loop poles is dominant and has a relative damping 1 . Correspondingly, the closed-loop system bandwidth is 1 (in terms of the dimensionless frequency introduced before). The former closed-loop pole is "far-away" relative to the dominant pair; it is there because of our insistence that the controller be proper.

We observe that the design for the case where $p$ is small is just the design that would have been obtained by classical frequency domain design methods. The advantage of the proposed method is that it can also be applied in situations (involving plants with several right-half plane poles and zeros, for instance) that cannot very easily be handled by classical techniques.
TABLE I

SOLUTIONS FOR THE DOUBLE INTEGRATOR

\begin{tabular}{ccccc}
\hline$p$ & $\lambda$ & $\chi_{0}$ & $\chi_{1}$ & $\chi_{2}$ \\
\hline $10^{-6}$ & 1.01263 & 112.012 & 159.409 & 113.427 \\
$10^{-3}$ & 1.12881 & 11.0055 & 16.5599 & 12.4232 \\
1 & 2.39652 & 0.976371 & 2.17791 & 2.33990 \\
$10^{3}$ & 11.9037 & 0.0749231 & 0.375100 & 0.891868 \\
\hline
\end{tabular}
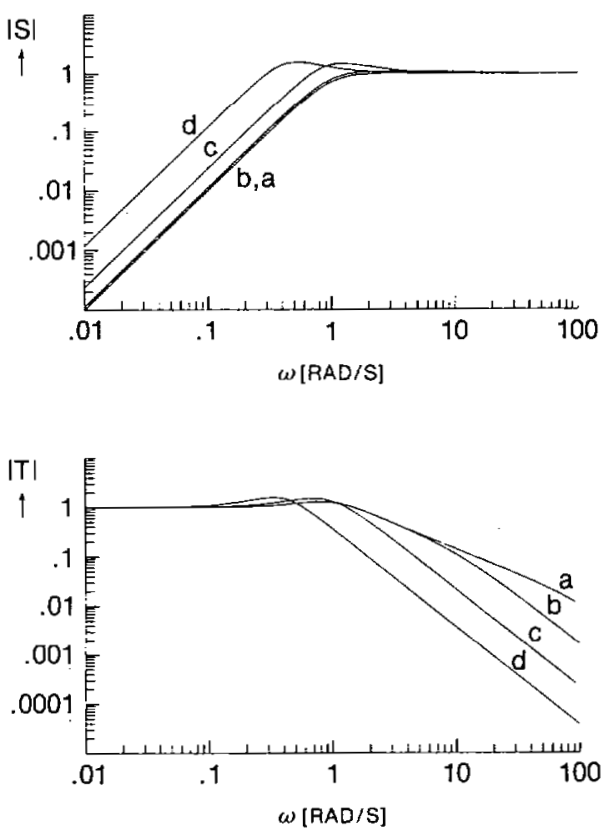

Fig. 2. Plots of optimal $|S|$ and $|T|$ for the double integrator: (a) $p=10^{-6}$, (b) $p=10^{-3}$, (c) $p=1$, (d) $p=10^{3}$

\section{CONCLUSIONS}

In this paper, various aspects of the performance of linear single-input single-output feedback systems are translated into required bounds on the sensitivity function $S$ and its complement $T$ on the imaginary axis. This leads to a frequency domain minimax optimization problem involving both these functions. The problem formulation is a generalization of that of Zames [6] and Zames and Francis [7]. It allows the inclusion of power and bandwidth constraints and permits handling plant poles and zeros on the imaginary axis.

The approach of the paper leads to a polynomial equation, whose solution yields the desired controller. Although it is clear that standard numerical techniques, such as the Newton-Raphson method, can be used for their solution, the question what is the best way of solving this equation for high-order systems needs further study. Another aspect that deserves more consideration is what flexibility there is in the choice of the weighting functions that occur in the optimization problem, and how modifications of the weighting functions affect the solution.

\section{APPENDIX A}

\section{PROOF OF THEOREM 1}

Theorem 1 concerns the existence of a solution to the equation (3.16). It is convenient to rewrite the equation in terms of $\mu:=1$ / $\lambda$. To this end, we define $\theta / \lambda=: \hat{\theta}, \zeta / \lambda=: \hat{\xi}, \hat{\pi}_{\mu}:=\pi_{1 / \mu}$, replace $\chi$ with $\hat{\chi}$ (to emphasize that we now take $\mu$ as the variable rather 
than $\lambda)$, and rewrite the relations (3.8), (3.10), and (3.16) as

$$
\begin{gathered}
\beta_{1} \phi_{+} \hat{\theta}+\beta_{2} \psi_{+} \hat{\xi}=\mu \hat{\chi}, \\
\alpha_{1} \alpha_{1}^{*} \beta_{2}^{*} \phi_{+} \hat{\theta}-\alpha_{2} \alpha_{2}^{*} \beta_{1}^{*} \psi_{+} \hat{\xi}=\hat{\pi}_{\mu} \hat{\chi}^{*} . \\
\alpha_{1} \alpha_{1}^{*} \phi_{+} \phi_{+}^{*} \hat{\theta} \hat{\theta^{*}}+\alpha_{2} \alpha_{2}^{*} \psi_{+} \psi_{+}^{*} \hat{\xi} \xi^{*}=\hat{\chi} \hat{\chi}^{*} .
\end{gathered}
$$

In the remainder of this Appendix for typographical simplicity all circumflexes are suppressed. We first consider the existence of a solution to (A.3) rather than (A.2).

Lemma A.I: For $|\mu|$ sufficiently small, the polynomial equations (A.3) and (A.1) have a unique family of solutions $\chi_{\mu}$, $\theta_{\mu}, \zeta_{\mu}$ with deg $\left(\chi_{\mu}\right)=x, \operatorname{deg}\left(\theta_{\mu}\right) \leq t, \operatorname{deg}\left(\zeta_{\mu}\right) \leq z$, whose coefficients depend continuously on $\mu$, such that $\chi_{\mu}$ is monic and has all its roots in the open left-half plane.

Proof: We first establish that the equation (A.3) has a solution as desired at $\mu=0$ and then use the implicit function theorem to establish its existence for $|\mu|$ sufficiently small. For $\mu$ $=0$, (A.1) reduces to $\beta_{1} \phi_{+} \theta+\beta_{2} \psi_{+} \zeta=0$. Since by the various assumptions $\beta_{1} \phi_{+}$and $\beta_{2} \psi_{+}$, have no common roots, this equation has the general solution $\theta=\beta_{2} \psi_{+} a, \xi=-\beta_{1} \phi_{+} a$, with $a$ an arbitrary polynomial. Substitution into (A.3) yields

$$
\begin{aligned}
\chi \chi^{*} & =a a^{*} \phi_{+} \phi_{+}^{*} \psi_{+} \psi_{+}^{*}\left(\beta_{1} \beta_{1}^{*} \alpha_{2} \alpha_{2}^{*}+\beta_{2} \beta_{2}^{*} \alpha_{1} \alpha_{1}^{*}\right) \\
& =a a^{*} \phi_{+} \phi_{+}^{*} \psi_{+} \psi_{+}^{*} \pi_{0} \pi_{0}^{*} .
\end{aligned}
$$

We see that this equation indeed has a solution such that $\chi$ has degree $x$, has all its roots in the open left-half plane and is monic, given by $\chi_{0}=a \phi_{+}^{*} \psi * \pi_{0}$, where $a$ is a constant such that $\chi_{0}$ is monic. It follows that $\theta_{0}=a \beta_{2} \psi_{+}, \zeta_{0}=-a \beta_{1} \phi_{+}$.

We now apply the implicit function theorem [22] to the set of equations for the coefficients of the polynomials $\chi, \theta$, and $\zeta$ that results when the polynomial equations (A.1) and (A.3) are expanded in powers of $s$ and the coefficients of like powers are equated. It is easily verified that (A.1) and (A.3) in this way yield the same number of equations as unknown coefficients if we look for a solution $\chi, \theta, \zeta$ of degrees, respectively $x, t$, and $z$, with $\chi$ monic. It follows from the implicit function theorem that these equations have a solution for $|\mu|$ sufficiently small, which is continuously dependent on $\mu$, if the Jacobian of these equations with respect to the unknown coefficients is nonsingular at the solution that is obtained at $\mu=0$. This Jacobian is nonsingular if and only if the homogeneous set of equations

$$
\begin{gathered}
\beta_{1} \phi_{+} \tilde{\theta}+\beta_{2} \psi+\tilde{\xi}=0, \\
\alpha_{1} \alpha_{1}^{*} \phi_{+} \phi_{+}^{*}\left(\theta_{0} \tilde{\theta}^{*}+\theta_{0}^{*} \tilde{\theta}\right)+\alpha_{2} \alpha_{2}^{*} \psi_{+} \psi_{+}^{*}\left(\zeta_{0} \tilde{\zeta}^{*}+\zeta_{0}^{*} \tilde{\zeta}\right)-\left(\chi_{0} \tilde{\chi}^{*}+\chi_{0}^{*} \tilde{\chi}\right)
\end{gathered}
$$$$
=0 \text {, }
$$

with $\operatorname{deg}(\tilde{\chi})=x-1, \operatorname{deg}(\tilde{\theta})=t, \operatorname{deg}(\tilde{\zeta})=z$, has the unique solution $\tilde{\chi}=0, \tilde{\theta}=0, \tilde{\xi}=0$. Here $\operatorname{deg}(\tilde{\chi})=x-1$ because we are looking for a solution such that $\chi$ is monic. The first of the equations (A.5) has the general solution $\tilde{\theta}=\beta_{2} \psi+c, \tilde{\xi}=-$ $\beta_{1} \phi_{+} c$, with $c$ an arbitrary polynomial. Since $\operatorname{deg}(\zeta)=z$ and also $\operatorname{deg}\left(\beta_{1} \phi_{+}\right)=z, c$ is necessarily a constant. Substituting these solutions into the second of the equations (A.5), we obtain after rearrangement

$$
\phi_{+}^{*} \psi_{+}^{*} \pi_{0}\left(\tilde{x}-c \phi_{+}^{*} \psi_{+}^{*} \pi_{0}\right)^{*}+\phi_{+} \psi_{+} \pi_{0}^{*}\left(\tilde{x}-c \phi_{+}^{*} \psi_{+}^{*} \pi_{0}\right)=0 .
$$

Now, if $a$ is a polynomial that has no common roots with $a^{*}$, the polynomial equation $a x+a^{*} x^{*}=0$ has the general solution $x=$ $a^{*} \omega$, where $\omega$ is any polynomial such that $\omega^{*}=-\omega$. As a result it follows from (A.6) that $\bar{\chi}-c \phi_{+}^{*} \psi * \pi_{0}^{*}=\phi_{+}^{*} \psi_{+}^{*} \pi_{0} \omega$, where $\omega$ is any polynomial such that $\omega^{*}=-\omega$. It follows that $\tilde{\chi}=(c+$ $\omega) \phi_{+}^{*} \psi_{+}^{*} \pi_{0}$. Since $\operatorname{deg}(\tilde{x})=x-1$ and $\operatorname{deg}\left(\phi_{+}^{*} \psi_{+}^{*} \pi_{0}\right)=x$, necessarily $\tilde{\chi}=0, c+\omega=0$, so that $\omega=-c$. From $\omega^{*}=-\omega$ it follows that $c=0, \omega=0$, which proves that (A.5) has the unique solution $\tilde{\chi}=0, \tilde{\theta}=0, \tilde{\zeta}=0$.
It follows that (A.3) has a solution for $|\mu|$ small enough such that $\chi_{\mu}$ is monic. Since the coefficients of $\chi_{\mu}$ vary continuously with $\mu$, and $\chi_{0}$ has all its roots in the open left-half plane, the roots of $\chi_{\mu}$ remain in the open left-half plane for $|\mu|$ sufficiently small. This completes the proof of Lemma A.1.

Proof of Theorem la): To prove part a) of Theorem 1 we shall first prove (A.2). This equation is obtained by factoring

$$
\begin{aligned}
& \left(\alpha_{1} \alpha_{1}^{*} \beta_{2}^{*} \phi_{+} \theta-\alpha_{2} \alpha_{2}^{*} \beta_{1}^{*} \psi_{+} \zeta\right)\left(\alpha_{1} \alpha_{1}^{*} \beta_{2}^{*} \phi_{+} \theta-\alpha_{2} \alpha_{2}^{*} \beta_{1}^{*} \psi_{+} \zeta\right)^{*} \\
& \quad=\pi_{\mu} \pi_{\mu}^{*} \chi \chi^{*}
\end{aligned}
$$

[compare (3.13)], which is equivalent to $Z(s)=\lambda^{2}$. Thus, given a solution of (A.2), (A.3) holds. We first prove that (A.2) is the correct factorization of (A.7). To this end, define $q_{\mu}:=$ $\alpha_{1} \alpha_{1}^{*} \beta_{2}^{*} \phi_{+} \theta_{\mu}-\alpha_{2} \alpha_{2}^{*} \beta_{1}^{*} \psi_{+} \zeta_{\mu}$. Then (A.7) takes the form $q q^{*}=$ $\pi_{\mu} \pi_{\mu}^{*} \chi \chi^{*}$, and (A.2) becomes $q=\pi_{\mu} \chi^{*}$. We thus have to prove that $q=\pi_{\mu} \chi^{*}$ is the correct factorization of $q q^{*}=\pi_{\mu} \pi_{\mu}^{*} \chi \chi^{*}$.

To this end, let the pair of polynomials $g$ and $h$ be any solution of the equation $\beta_{1} \phi_{+} g+\beta_{2} \psi_{+} h=1$. By the various assumptions, $\beta_{1} \phi_{+}$and $\beta_{2} \psi_{+}$are relatively prime and a solution $g, h$ exists. Then given $\mu \chi,(\mathrm{A} .1)$ has the general solution

$$
\theta=g \mu \chi+\beta_{2} \psi+\omega, \zeta=h \mu \chi-\beta_{1} \phi_{+} \omega
$$

where $\omega$ is an arbitrary polynomial. Substitution of these expressions for $\theta$ and $\zeta$ into (A.3) yields an equation of the form

$$
\begin{aligned}
0= & -\chi^{*} \delta_{11} \chi+\mu \chi^{*} \phi_{+} \psi_{+} \delta_{12}^{*} \omega+\mu \chi \phi_{+}^{*} \psi_{+}^{*} \delta_{12} \omega^{*} \\
& +\omega^{*} \phi_{+} \phi_{+}^{*} \psi_{+} \psi_{+}^{*} \pi_{0} \pi_{0}^{*} \omega,
\end{aligned}
$$

where $\delta_{11}:=1-\mu^{2}\left(\alpha_{1} \alpha_{1}^{*} \phi_{+} \phi_{+}^{*} g g^{*}+\alpha_{2} \alpha_{2}^{*} \psi_{+} \psi_{+}^{*} h h^{*}\right), \delta_{12}:=$ $\alpha_{1} \alpha_{1}^{*} \phi_{+} \beta_{2}^{*} g-\alpha_{2} \alpha_{2}^{*} \psi_{+} \beta_{1}^{*} h$. Similarly, using (A.8) it follows from the definition of $q$ that

$$
q=\mu \delta_{12} \chi+\pi_{0} \pi_{0}^{*} \phi_{+} \psi+\omega
$$

Comparison of (A.9) and (A.10) reveals that $0=\chi^{*}\left(-\delta_{11} \chi+\right.$ $\left.\mu \phi_{+} \psi_{+} \delta_{12}^{*} \omega\right)+q_{\mu} \phi_{+}^{*} \psi_{+}^{*} \omega^{*}$, which we rewrite in the form

$$
q \phi_{+}^{*} \psi_{+}^{*} \omega^{*}=\chi^{*} \epsilon
$$

where $\epsilon$ is the polynomial $\epsilon:=\mu \phi_{+} \psi_{+} \delta_{12}^{*} \omega-\delta_{11} \chi$. Now, since $\chi$ is stable for $\mu$ sufficiently small, $\chi$ has no roots in common with $\phi_{+}$and $\psi_{+}$. Furthermore, for $\mu=0$ we have $\omega_{0}=a, \chi_{0}=$ $a \phi * \psi * * \pi_{0}$ (see the proof of Lemma A.1) so that $\omega_{0}$ and $\chi_{0}$ have no common roots; therefore, $\chi$ and $\omega$ have no common roots for $\mu$ sufficiently small. Because as a result $\chi^{*}$ has no roots in common with $\phi_{+}^{*} \psi_{+}^{*} \omega^{*}$, it follows from (A.11) that any root of $\chi^{*}$ is a root of $q$, which means that $q$ has a factor $\chi^{*}$.

From this we conclude that $q=\bar{\pi} \chi^{*}$, with $\bar{\pi}$ a polynomial to be determined. From $q q^{*}=\pi_{\mu} \pi_{\mu}^{*} \chi \chi^{*}$ it follows that $\bar{\pi}^{*}=\bar{\pi}_{\mu} \bar{\pi}_{\mu}^{*}$. Now, from the definition of $q$ it easily follows that $q_{0}=$ $a \pi_{0} \pi_{0}^{*} \phi_{+} \psi_{+}$, while from $q=\bar{\pi} \chi^{*}$ we obtain $q_{0}=a \bar{\pi}_{0} \pi_{0}^{*} \phi_{+} \psi_{+}$, which shows that $\bar{\pi}_{0}=\pi_{0}$. This implies that for $\mu=0$, and hence for $\mu$ sufficiently small, $\bar{\pi}$ is stable. Therefore, the correct factorization of $\bar{\pi} \bar{\pi}^{*}=\pi_{\mu} \pi_{\mu}^{*}$ is $\bar{\pi}=\pi_{\mu}$, which proves that $q=$ $\pi_{\mu} \chi^{*}$ as claimed. Having proved the validity of (A.2), we now consider (A.2) together with (A.1) as equations for the unknown polynomials $\theta, \zeta$, and $\chi$. As has been shown, there exists a solution for $\mu$ small enough, which is unique within multiplication by a continuous function of $\mu$.

We now observe that for $\mu$ given $\pi_{\mu}$ is known, and hence (A.2) and (A.1) form a set of homogeneous linear equations in the coefficients of the unknown polynomials $\chi, \theta$, and $\zeta$. A coefficients-versus-equations count reveals that this linear set of equations is overdetermined (unless $b_{1}=b_{2}=e=0$; then we have as many equations as unknowns). Let us arrange the unknown coefficients of the polynomials $\chi, \theta$, and $\zeta$ (not restricting $\chi$ to be monic) in a vector $y$; then the equations (A.2) and (A.1) can be put into the form $A_{\mu} y=0$, where $A_{\mu}$ is a 
rectangular matrix. This equation has a nontrivial solution if and only if $A_{\mu}^{\prime} A_{\mu}$ (with the prime denoting the transpose) is singular. Since a nontrivial solution exists for $\mu$ small enough, it follows that det $\left(A_{\mu}^{\prime} A_{\mu}\right)=0$ for $\mu$ small enough. Now the elements of $A_{\mu}$ are analytic functions of the coefficients of $\pi_{\mu}$, and these coefficients are analytic functions of $\mu$ for $|\mu|<1 / \lambda_{o}$, so that det $\left(A_{\mu}^{\prime} A_{\mu}\right)$ is an analytic function of $\mu$ for $|\mu|<1 / \lambda_{o}$. It follows that $\operatorname{det}\left(A_{\mu}^{\prime} A_{\mu}\right)=0$ for $|\mu| \leq 1 / \lambda_{a}$, and hence that (A.2) and (A.1) have a nontrivial solution with the properties stated in Theorem (a) for $|\mu| \leq 1 / \lambda_{0}$.

Proof of Theorem $1 b$ ): Suppose that $\chi_{1 / \lambda_{0}}$ has a root in the open right-half plane. If $\chi-1 / \lambda_{o}$ has such a root, the proof follows similarly. We track the solution of (A.2) as $\mu$ is increased from 0 to $1 / \lambda_{o}$. Since the coefficients of $\chi_{\mu}$ vary continuously with $\mu$, and for $\mu$ small enough all roots of $\chi_{\mu}$ are in the open left-half plane but by hypothesis for $\mu=1 / \lambda_{o}$ at least one root of $\chi_{\mu}$ is the open right-half plane, for some $\mu<1 / \lambda_{o}$ at least one root of $\chi_{\mu}$ crosses over to the right-half plane, either via infinity or via the imaginary axis.

Let us consider what happens to the polynomials $\theta$ and $\zeta$. Solving (A.1) and (A.2) for $\phi_{+} \theta$ and $\psi_{+} \zeta$ yields

$$
\left[\begin{array}{c}
\phi_{+} \theta \\
\psi_{+} \zeta
\end{array}\right]=\frac{1}{\pi_{0} \pi_{0}^{*}}\left[\begin{array}{cc}
\alpha_{2} \alpha_{2}^{*} \beta_{1}^{*} & \beta_{2} \\
\alpha_{1} \alpha_{1}^{*} \beta_{2}^{*} & -\beta_{1}
\end{array}\right]\left[\begin{array}{c}
\mu \chi \\
\pi_{\mu} \chi^{*}
\end{array}\right] .
$$

Suppose that a root of $\chi_{\mu}$ crosses over via infinity, so that $\chi_{\mu}$ loses degree. Then also $\chi_{\mu}^{*}$ loses degree and (A.12) shows that $\theta_{\mu}$ and $\zeta_{\mu}$ simultaneously lose degree as well; clearly we have a solution as indicated in Theorem 1b). Suppose on the other hand that a root of $\chi_{\mu}$ reaches the imaginary axis. Then by conjugate symmetry also $\chi_{\mu}^{*}$ has this root, so that $\chi_{\mu}$ and $\chi_{\mu}^{*}$ have a common root on the imaginary axis. By (A.12), $\theta_{\mu}$ and $\zeta_{\mu}$ also have this root (note that by assumption c) $\pi_{0}$ has no roots on the imaginary axis so that it is impossible that the common root of $\theta_{\mu}$ and $\zeta_{\mu}$ is canceled by a root of $\pi_{0}$ or $\pi_{0}^{*}$ ). Thus, $\chi_{\mu}, \theta_{\mu}$, and $\zeta_{\mu}$ have a common root on the imaginary axis, which can be canceled throughout in the equations (A.1) and (A.2), again leading to a solution of reduced degree. This completes the proof of Theorem 1b).

\section{APPENDIX B}

\section{Proof of lemMa 2}

In this Appendix the details of the last step of the proof of Lemma 2 are supplied. Suppose first that $\gamma-\eta / \lambda^{2}$ loses degree at $\lambda_{o}$ and that $\gamma-\eta / \lambda^{2}$ has no roots on the imaginary axis for any $|\lambda| \geq \lambda_{o}$. Then $\gamma(i \omega)-\eta(i \omega) / \lambda^{2}>0$ for all $|\lambda| \geq \lambda_{o}$ and all $\omega$. Since the leading coefficient of $\gamma-\eta / \lambda^{2}$ changes sign at $\lambda_{o}$, there exists a $|\lambda|<\lambda_{o}$ such that $\gamma(i \omega)-\eta(i \omega) / \lambda^{2}<0$ for $\omega$ large enough. It follows that inf $\left\{\lambda^{2}: \gamma(i \omega)-\eta(i \omega) / \lambda^{2} \geq 0\right.$ for all $\left.\omega\right\}$ $=\lambda_{o}^{2}$. Next, suppose that $\lambda_{o}$ is the first value of $\lambda$ such that $\gamma-$ $\eta / \lambda^{2}$ has a root on the imaginary axis as $\lambda$ is decreased from $\infty$, and suppose that this root is $i \omega_{0}$. Then $\gamma(i \omega)-\eta(i \omega) / \lambda^{2} \geq 0$ for all $|\lambda| \geq \lambda_{o}$ and all $\omega$. Now, the derivative of $\gamma-\eta / \gamma^{2}$ with respect to $1 / \lambda^{2}$ at $s=i \omega_{o}$ equals $-\eta\left(i \omega_{o}\right)$. Since $\gamma\left(i \omega_{o}\right)-\eta(i \omega) /$ $\lambda^{2}=0$ and [by assumption c)] $\gamma\left(i \omega_{o}\right)>0$ it follows that $\eta\left(i \omega_{o}\right)$ $>0$; hence there exist a $|\lambda|<\lambda_{o}$ such that $\gamma\left(i \omega_{o}\right)-\eta\left(i \omega_{o}\right) / \lambda^{2}<$ 0 . It follows again that inf $\left\{\lambda^{2}: \gamma(i \omega)-\eta(i \omega) / \lambda^{2} \geq 0\right.$ for all $\left.\omega\right\}$ $=\lambda_{o}^{2}$.

\section{APPENDIX C}

\section{ProOf of LEMMA 4}

The proof of this lemma is a standard application of Wiener optimization theory [23]-[25]. For a plant $H=\psi / \phi$ and a controller $G=\rho / \sigma$, the closed-loop characteristic polynomial is

$$
\chi_{c l}=\phi \sigma+\psi \rho .
$$

Let the polynomials $\xi$ and $\eta$ solve the diophantine equation $\phi \xi+$ $\psi \eta=1$ (since by assumption $\phi$ and $\psi$ have no common roots a solution exists). Then for given $\chi_{c l}$, (C.1) can be solved for $\sigma$ and .$\rho$ in the form

$$
\sigma=\xi \chi_{c l}+\psi \omega, \rho=\eta \chi_{c l}-\phi \omega
$$

with $\omega$ an arbitrary polynomial. Accordingly, we obtain for the sensitivity function and its complement

$$
S=\frac{\phi \sigma}{\chi_{c l}}=\phi \xi+\phi \psi P, T=\frac{\psi \rho}{\chi_{c l}}=\psi \eta-\phi \psi P
$$

where $P:=\omega / \chi_{c l}$. This is a well-known parametrization of $S$ and $T$ in terms of the rational function $P$. If the closed-loop system is required to have all its poles in the closed left-half plane, $P$ can range over all rational functions having their poles in the closed left-half plane.

Define $\mathcal{K}$ as the set of rational functions $P$ with all their poles in the closed left-half plane such that $V S$ and $W T$ are proper and have no poles on the imaginary axis. Without loss of generality we limit the class of admissible control systems to those for which $P$ $\in \mathcal{K}$. Now, consider two control systems, respectively parametrized by $P \in \mathfrak{K}$ and $\tilde{P} \in \mathcal{K}$. Then using (C.3) it is not difficult to find that one can write

$$
\begin{aligned}
\tilde{Z}= & V V^{*} S S^{*}+W W^{*} T T^{*}+\left(V V^{*} S-W W^{*} T\right)^{*} \phi \psi(\tilde{P}-P) \\
& +\left(V V^{*} S-W W^{*} T\right) \phi^{*} \psi^{*}(\tilde{P}-P)^{*} \\
& +\left(V V^{*}+W W^{*}\right) \phi \phi^{*} \psi \psi^{*}(\tilde{P}-P)(\tilde{P}-P)^{*}
\end{aligned}
$$

Here, in an obvious notation, $\tilde{Z}$ corresponds to the control system parametrized by $\tilde{\boldsymbol{P}}$, and $S$ and $T$ to that parametrized by $\boldsymbol{P}$. A standard variational argument shows that if an optimal solution exists, the control system parametrized by $P$ maximizes the auxiliary criterion $(3.18)$ if and only if

$$
\int_{-\infty}^{\infty}(\tilde{P}-P) \Sigma \phi \psi\left(|V|^{2} S-|W|^{2} T\right)^{*} d \omega=0
$$

for all $\tilde{P} \in \mathfrak{K}$. Since for $P$ and $\tilde{P}$ in $\mathscr{K}$ the rational function $(\tilde{P}-$ $P) \phi \psi\left(V V^{*} S^{*}-W W^{*} T^{*}\right)$ has no poles on the imaginary axis and is proper, a simple application of the residue theorem shows that a sufficient condition for (C.5) to hold is that $\Sigma \phi \psi\left(V V^{*} S^{*}-\right.$ $W W^{*} T^{*}$ ) has all its poles in the closed left-half plane. To establish uniqueness, we observe by inspection of (C.4) using (C.5) that the control system parametrized by $\tilde{P}$ is optimal if and only if

$$
\int_{\infty}^{\infty} \Sigma\left(|V|^{2}+|W|^{2}\right)|\phi|^{2}|\psi|^{2}|\tilde{P}-P|^{2} d \omega=0
$$

Since by assumption $\Sigma$ is positive for all $\omega$ this implies that $\tilde{P}=P$ and hence the optimal solution is unique.

This proof is due to $H$. Westdijk [26].

\section{REFERENCES}

[1] J. C. Doyle, "Robustness of multiloop linear feedback systems," in Proc. 17th IEEE Conf. Decision Contr., 1979, pp. 12-18.

[2] J. C. Doyle and G. Stein, "Multivariable feedback design: Concepts for a classical/modern synthesis," IEEE Trans. Automat. Contr., vol. AC-26, pp. 4-16, 1981.

[3] J. C. Doyle, "Achievable performance in multivariable feedback systems," in Proc. 18th IEEE Conf. Decision Contr., 1979, pp. $250-251$.

[4] - "Limitations on achievable performance of multivariable feedback systems," in Multivariable Analysis and Design-Techniques (Agard Lecture Series 117) 1981.

[5] J. C. Doyle, J. E. Wall, and G. Stein, "Performance and robustness analysis for structured uncertainty," in Proc. 21st IEEE Conf. Decision and Contr., 1982.

[6] G. Zames, "Feedback and optimal sensitivity: Model reference 
transformations, multiplicative seminorms, and approximate inverses," IEEE Trans. Automat. Contr., vol. AC-26, pp. 301-320, 1981 (previously presented at the 1979 Allerton Conf.).

[7] G. Zames and B. A. Francis, "Feedback, minimax sensitivity, and optimal robustness," IEEE Trans. Automat. Contr., vol. AC-28, pp. $585-601,1983$.

[8] B. Francis and G. Zames, "On $\mathfrak{F} \mathcal{C}^{\infty}$-optimal sensitivity theory for SISO feedback systems," IEEE Trans. Automat. Contr., vol. AC-29, pp. 9-16, 1984 .

[9] B.-C. Chang and J. B. Pearson, "Optimal disturbance reduction in linear multivariable systems," in Proc. IEEE Conf. Decision Contr., San Antonio, TX, Dec. 1983.

[10] B. A. Francis, J. W. Helton, and G. Zames, " $\mathrm{H}^{\infty}$-optimal feedback controllers for linear multivariable systems," IEEE Trans. Automat. Contr., vol. AC-29, pp. 880-900, 1984.

[11] M. G. Safonov, A. J. Laub, and G. L. Hartmann, "Feedback properties of multivariable systems: The role and use of the return difference matrix," IEEE Trans. Automat. Contr., vol. AC-26, pp. $47-65,1981$.

[12] H. Kwakernaak, "Minimax frequency domain optimization of multivariable linear feedback systems," in Proc. 1984 IFAC World Congr., Budapest, Hungary, 1984.

[13] H. Kwakernaak, "Robustness optimization of linear feedback systems," in Proc. 22nd IEEE Conf. Decision Contr., San Antonio, TX, Dec. 1983.

[14] $M$. Verma and $E$. Jonckheere, " $L{ }^{\infty}$-compensation with mixed sensitivity as a broadband matching problem,"' Syst. Contr. Lett., vol. 4, pp. $125-129,1984$

[15] J. W. Helton, "Worst case analysis in the frequency domain: the $\mathbf{H}^{\propto}$ approach to control," in Proc. IEEE Conf. Decision Contr., San Antonio, TX, Dec, 1983.

[16] B. A. Francis, "Optimal disturbance attenuation with control weighting," in Proc. Twente Workshop on Systems and Optimization, LNCIS vol. 66, A. Bagchi and H. Th. Jongen, Eds. Berlin: SpringerVerlag, 1984

[17] P. Henrici, Applied and Computational Complex Analysis, Vol. i. New York: Wiley, 1974.

[18] S. Zaks, The Theory of Statistical Inference. New York: Wiley, 1971

[19] V. Kucera, Discrete Linear Control. New York: Wiley, 1979.

[20] S. Smulders, "Two computer programs for computing minimax frequency domain optimal controllers," Dep. Appl. Math., Twente Univ. Technol.. Memo. 1984.
[21] Th. Kailath, Linear Systems. Englewood Cliffs, NJ: Prentice-Hall, 1980.

[22] J. Dieudonné, Foundations of Modern Analysis. New York: Academic, 1960.

[23] D. C. Youla, J. J. Bongiorno, and H. A. Jabr, "Modern Wiener-Hopf design of optimal controllers-Part I," IEEE Trans. Automat. Contr., vol. AC-21, pp. 3-15, 1976.

[24] " "Modern Wiener-Hopf design of optimal controllers-Part II," IEEE Trans. Automat. Contr., vol. AC-21, pp. 319-338, 1976.

[25] C. A. Desoer, R. W. Liu, J. Murray, and R. Saeks, "Feedback system design: The fractional representation approach to analysis and synthesis," IEEE Trans. Automat. Contr., vol. AC-25, pp. 401-412, 1980.

[26] H. Westdijk, "Optimale robuustheid van lineaire regelsystemen," Dep. Appl. Math., Twente Univ. Technol., Enschede, The Netherlands, Student Project Rep., May 1983.

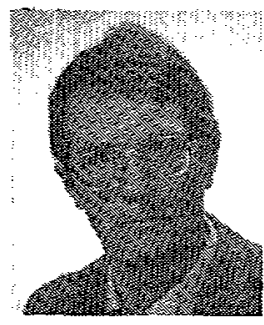

Huibert Kwakernaak (S'61-M'63-SM'72-F'83) was born in Rijswijk (Z.H.), The Netherlands, on March 18, 1937. He received the final diploma in engineering physics from Delft University of Technology, Delft, The Netherlands, in 1960, and the M.Sc. and Ph.D. degrees in electrical engineering from the University of California at Berkeley in 1962 and 1963, respectively.

Since 1964 he has held a succession of positions in the Departments of Engineering Physics and Mathematics, Delft University of Technology. In 1970 he was appointed Professor in the Department of Applied Mathematics, Twente University of Technology, Enschede, The Netherlands. He has published about 50 papers in the area of systems and control. He is the author, with R. Sivan, of the book Linear Optimal Control Systems (New York: Wiley-Interscience, 1972) and, with H. W. Knobloch, of the book Lineare Kontrolltheorie (New York: Springer-Verlag, 1985). His current interests are in linear control theory.

Dr. Kwakernaak has held several positions in IFAC and is Editor of the IFAC Journal Automatica. 